\title{
Blood and Lymph Circulating Cells: Well-Known Systems, Well-Forgotten Interdependence
}

\section{Ekaterina I. Galanzha*}

Philips Classic Laser \& Nanomedicine Laboratories, University of Arkansas for Medical Sciences, 4301 West Markham St., Slot 543, Little Rock, Arkansas 72205-7199, USA

Cells are basic unit of life, and their diagnosis has already revolutionized many branches of biology and medicine. When cells are functioning in a whole organism, they often move through the body and their dissemination play key role in health and diseases. For instance, the deadly progression of cancers, infections and cardiovascular diseases, leading to more deaths ( 65\%) than other causes in the world, are mainly a result of cell dissemination including circulating tumor cells (CTCs) as the roots of cancer metastasis; bacteria as causes of infectious sepsis; and platelets as the important participants of heart attacks and strokes [1-6].

The facts that common pathways for cell dissemination are blood and lymph vessels and those cells may pass from one system to another through numerous anatomical interconnections between lymph vessels, lymph nodes and blood circulatory system have been well known for many years $[7,8]$.

The close interrelations of blood and lymph systems can be illustrated by simplified schematic of dissemination of CTCs in cancer (Figure 1). Logically, to understand disease progression, both lymph and blood pathways should be examined simultaneously. Nevertheless, until now, cells (e.g., CTCs) in blood and lymph have been studied separately, with a particular focus on the role of blood cells. This skewed perspective cannot provide a comprehensive understanding of the mechanisms of disease development. As a result, we currently do not have the clinical capability to intervene and stop development of many diseases at early stage when well-timed treatment is most effective.

Furthermore, the major obstacle for assessment of cells in lymph flow is that lymph is a challenging substance to study [9-11]. Compared with blood vasculature, lymph vessels are colorless, with relatively low pressure and low concentrations of cells. As a result, lymph sampling is impractical because it yields only a few microliters at a time and requires long-term cannulation.

The reasonable solution to detect cells moving in both, lymph and blood vascular, systems is using in vivo approaches. Among different in vivo methods (e.g., MRI, PET, or optical assays), in vivo flow cytometry, using multiphoton detection of cells labeled in vivo

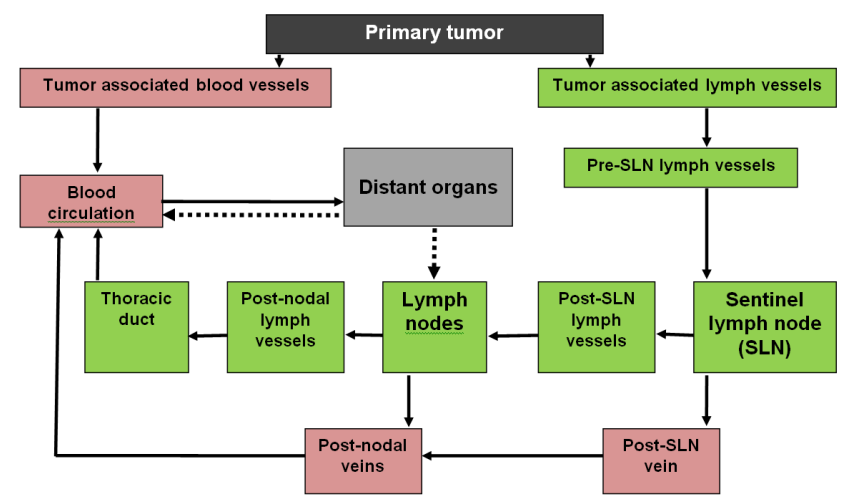

Figure 1: Pathways of CTCs from primary tumor to metastatic sites. by fluorescent conjugates (e.g., folate for breast cancer CTCs) has demonstrated the highest sensitivity. For example, a concentration the threshold for detection of CTCs in a mouse model was estimated at $\sim 2$ CTCs/mL $[12,13]$. Despite the contribution of this work, the use of fluorescent labeling in vivo raises potential problems for translating this technology to humans because of: (1) the cytotoxicity of available fluoresecent tags; (2) the strong immune response to tags; and (3) the effects of light scattering and the autofluorescent background, which allow the assessment of only superficial microvessels with a slow flow rate, significantly lengthening the examination of large blood volumes (up to few days) [14-16]. Recently developed photoacoustic (PA) and photothermal (PT) flow cytometry (PAFC and PTFC, respectively) represents an advanced alternative for the detection of individual blood and lymph cells in vivo because PAFC and PTFC are not sensitive to light scattering and autofluorescent phenomena and they provide higher resolution at deeper tissue (at depths up to $3-5 \mathrm{~cm}$ ) assessments as compared to other optical modalities [10,14]. Experimental results demonstrated that PAFC has the unprecedented sensitivity threshold, as one CTC in the background of 106 WBCs, for in vivo lymph testing $[10,15,16]$ with potential to achieve a 100 -fold gain in sensitivity in blood (i.e., $1 \mathrm{CTC} / 100 \mathrm{~mL}$ ). In addition, high-speed PAFC may detect fast-moving CTCs flowing through the detection point at 10-3-10-4 $\mathrm{sec}$, and, thus, it may provide rapid monitoring of an almost entire blood volume during $\sim 1$ hour for humans because 3-5 L of blood passes through peripheral blood vessels with diameters of 2-3 $\mathrm{mm}$ and a typical flow rate of $100 \mathrm{ml} / \mathrm{min}$ [17]. The important advantages of PAFC versus the other existing technologies include noninvasive realtime enumeration and differentiation of morphological and functional types of cells in circulating blood or lymph (e.g., CTCs, bacteria, clots and white blood cells [WBCs]) based on their multiplex molecular targeting that can combined with intravascular magnetic enrichment and targeted eradication of pathological cells (e.g., CTCs) [10,14-20].

Recently we integrated blood and lymph PAFC and demonstrated its application with PA lymphography, PA cytometry in solid organs, using functionalized nanoparticles (NPs) as high-contrast low-toxic PA and PT contrast agents $[15,16,21]$. Such advanced technical platform provided simultaneous real-time quantitative monitoring of blood and lymph CTCs in vivo and define in vivo cross-correlations between

*Corresponding author: Ekaterina I. Galanzha, Philips Classic Laser \& Nanomedicine Laboratories, University of Arkansas for Medical Sciences, 4301 West Markham St., Slot 543, Little Rock, Arkansas 72205-7199, USA, Tel.: +501526-7620; Fax: +501-686-8029; E-mail: Galanzha@uams.edu

Received December 16, 2011; Accepted December 17, 2011; Published December 18, 2011

Citation: Galanzha El (2011) Blood and Lymph Circulating Cells: WellKnown Systems, Well-Forgotten Interdependence. J Blood Lymph 1:e104. doi:10.4172/2165-7831.1000e104

Copyright: (C) 2011 Galanzha El. This is an open-access article distributed unde the terms of the Creative Commons Attribution License, which permits unrestricted use, distribution, and reproduction in any medium, provided the original author and source are credited. 
Citation: Galanzha El (2011) Blood and Lymph Circulating Cells: Well-Known Systems, Well-Forgotten Interdependence. J Blood Lymph 1:e104. doi:10.4172/2165-7831.1000e104

Page 2 of 2

\begin{tabular}{|l|l|l|l|l|l|}
\hline & $\begin{array}{l}\text { Tumor size, } \\
\text { mm2 }\end{array}$ & $\begin{array}{l}\text { Rate of lymph } \\
\text { CTCs, cell/ } \\
\text { min }\end{array}$ & $\begin{array}{l}\text { Rate of } \\
\text { blood CTCs, } \\
\text { cell/min }\end{array}$ & $\begin{array}{l}\text { Number of PA sig- } \\
\text { nals associated } \\
\text { with metastasis in } \\
\text { SLNs }\end{array}$ & Histology \\
\hline 1 week & $1.0 \pm 0.2$ & $0.26 \pm 0.05$ & $0.85 \pm 0.03$ & 493 & NO \\
\hline 2 weeks & $3.6 \pm 0.5$ & $2.13 \pm 0.30$ & $1.07 \pm 0.05$ & 3,188 & YES \\
\hline
\end{tabular}

Table 1: Correlation between primary tumor size, metastasis in SLN and number of tumor cells in lymph \& blood flow.

lymph CTCs, blood CTCs, size of primary tumor and nodal and distant metastases. The unprecedented sensitivity and specificity allowed studying CTC pathways at the earliest latent stage of metastatic disease. Specifically, in a preclinical mouse melanoma model, we found that early metastatic cells in latent metastatic disease (4-7 days after tumor inoculation) can equally disseminate by blood and lymph pathways (Table 1). However, in a few cases, metastatic cells appeared in lymph vessels in week 1 without any cells detected in the blood vessels and vice versa, suggesting individualized character of CTC dissemination. On average, over the course of two weeks extending area of primary tumor in $\sim 3.5$ times is associated with a increase in the quantity of lymphatic CTCs by 10 times, with only a little increase in blood CTCs but accompanied by 6.5 -fold increase in the number of PA signals (i.e., micrometastasis development) from the sentinel lymph nodes. In general, we showed that PAFC can overcome great challenge in cancer research related to the ability to test lymph and blood CTCs at the same time points at single-cell levels in the natural microenvironment in vivo over long-term period of metastatic disease development.

Because tumor cells can disseminate to distant sites during the earliest stage of tumor development [22], the ability to provide a dynamic count of DTCs in lymph and CTCs in blood should have great clinical relevance for cancer patients without overt metastasis and may open new avenues for early prognosis of metastasis and its successful individualized treatment. Taking into account the safe nature of the PAFC, it may be quickly translated to use in humans. The immediate clinical utility centers on an integrated, robust tool that would serve as a portable "personal" flow cytometer for in vivo, ultra-sensitive integrative lymph/blood cancer testing and could even enable new, highly targeted treatment modalities.

The great importance of studying blood and lymph is not limited by circulating cells. Better understanding physiological processes (e.g., immunity) and diseases requires detailed attention to monitoring lymph/blood chemistry (e.g., blood and lymph rheology), counting disease-associated small circulating objects (e.g., apoptotic and necrotic products, proteins, microparticles and DNA) and determining pharmacokinetics of drugs and NPs $[10,18,20,23]$.

Finally I want to emphasize that the studying blood and lymph on single-cell (particle) level is crucial, both for understanding basic cell biology (e.g. metabolism and apoptosis), cell flow dynamics, and for conducting clinical studies of early disease diagnoses (e.g., cancer, heart attack, stroke, sickle diseases, leukemia, edema, inflammation, infections) or assessment of innovative therapeutic interventions (pharmaceuticals, nicotine, lasers, or $\gamma$-radiation). I hope that these short editorial comments will help to attract the scientific interest and support that are required to solve these problems. I hope that the newborn Journal of Blood \& Lymph will facilitate research in these directions. Furthermore, I hope that the covering of the topics related to finding, counting, and killing rare but potentially deadly cells/objects in both the blood and lymphatic systems will be one of the top priorities of Journal of Blood \& Lymph. I am sure that the open assess of Journal of
Blood \& Lymph and, thus, quick dissemination of research knowledge in scientific media can catalyze transformation of the conventional medicine, wherein the disease is treated when symptoms appear and normal function is lost, to the advanced medicine, in which an intervention is applied before symptoms appear to preserve normal function for as long as possible.

\section{References}

1. Kochanek KD, Xu J, Murphy SL, Miniño AM, Kung H-C (2011) Deaths: Preliminary Data for 2009. National vital statistics report 59: 1-51.

2. Couzin J (2003) Tracing the steps of metastasis, cancer's menacing ballet Science 299: 1002-1006.

3. Christofori G (2006) New signals from the invasive front. Nature 441: 444-450.

4. Zhe X, Cher ML, Bonfil RD (2011) Circulating tumor cells: finding the needle in the haystack. Am J Cancer Res 1: 740-751.

5. Feigin VL, Lawes CM, Bennett DA, Barker-Collo SL, Parag V (2009) Worldwide stroke incidence and early case fatality reported in 56 population-based studies: a systematic review. Lancet Neurol 8: 355-369.

6. Ruggeri, Z.M. Platelets in atherothrombosis (2002) Nat Med. 8: 1227-1234.

7. Casley-Smith JR (1976) The functioning and interrelationships of blood capillaries and lymphatics. Experientia 32: 1-12.

8. Wiederhielm CA (1977) Blood-lymph transport mechanisms. Bibl Anat $15 \mathrm{Pt}$ 1: $477-482$.

9. Brown $P(2005)$ Lymphatic system: unlocking the drains. Nature 436: 456-458

10. Galanzha El, Shashkov EV, Tuchin VV, Zharov VP (2008) In vivo multiparameter, multispectral lymph flow cytometry with natural cell focusing, label-free detection and multicolor nanoparticle probes. Cytometry A 73: 884-894.

11. Olszewski W, Tarnok A (2008) Photoacoustic listening of cell in lymphatics: research art or novel clinical noninvasive lymph test. Cytometry A 73: 1111-1113.

12. He W, Wang H, Hartmann LC, Cheng JX, Low PS (2007) In vivo quantitation of rare circulating tumor cells by multiphoton intravital flow cytometry. Proc Nat Acad Sci 104: 11760-11765.

13. Hwu D, Boutrus S, Greiner C, DiMeo T, Kuperwasser C, et al. (2011) Assessment of the role of circulating breast cancer cells in tumor formation and metastatic potential using in vivo flow cytometry. J Biomed Opt 16: 040501.

14. Tuchin VV, Tárnok A, Zharov VP (2011) In vivo flow cytometry: A horizon of opportunities. Cytometry A 79: 737-745.

15. Galanzha El, Shashkov EV, Kelly T, Kim J-W, Yang L, et al. (2009) In vivo magnetic enrichment and multiplex photoacoustic detection of circulating tumour cells. Nature of Nanotechnology 4: 855-860.

16. Galanzha EI, Shashkov EV, Spring P, Suen JY, Zharov VP (2009) In vivo label-free detection of circulating metastatic melanoma cells by two-color photoacoustic flow cytometry. Cancer Res 69: 7926-7934.

17. Nedosekin DA, Sarimollaoglu M, Shashkov EV, Galanzha EI, Zharov VP (2010) Ultra-fast photoacoustic flow cytometry with a $0.5 \mathrm{MHz}$ pulse repetition rate nanosecond laser. Opt Express 18: 8605-8620.

18. Zharov VP, Galanzha El, Shashkov EV, Kim J-W, Khlebtsov NG, et al. (2007) Photoacoustic flow cytometry: principle and application for real-time detection of circulating single nanoparticles, pathogens, and contrast dyes in vivo. $J$ Biomed Opt 12: 051503.

19. Galanzha El, Sarimollaoglu M, Nedosekin DA, Keyrouz SG, Mehta JL, et al (2011) In vivo flow cytometry of circulating clots using negative photothermal and photoacoustic contrasts. Cytometry A 79: 814-824

20. Galanzha El, Zharov VP (2011) In vivo photoacoustic and phototherma cytometry for monitoring multiple blood rheology parameters. Cytometry A 79 : 746-757.

21. Galanzha El, Kokoska MS, Shashkov EV, Kim J-W, Zharov VP (2009) In vivo photoacoustic detection and photothermal purging of metastasis in sentinel lymph nodes. J Biophotonics 2: 528-539.

22. Klein CA (2009) Parallel progression of primary tumours and metastases. Na Rev Cancer 9: 302-312.

23. Alix-Panabières $\mathrm{C}$, Schwarzenbach $\mathrm{H}$, Pantel K (2011) Circulating Tumor Cells and Circulating Tumor DNA. Annu Rev Med Jan 26. [Epub ahead of print] 